\title{
Measurement of Complex Permeability Using Short Coaxial Line Reflection Method
}

\author{
V. RAdOniĆ*, N. BlaŽ AND LJ. ŽIVANOV \\ Faculty of Technical Sciences, University of Novi Sad, Trg Dositeja Obradovica 6, Novi Sad, Serbia
}

This paper describes realization of complex permeability determination for ferrite materials using short coaxial sample holder and vector network analyzer E5071B in frequency range between $300 \mathrm{kHz}$ and $1 \mathrm{GHz}$. The design of coaxial high frequency sample holder is presented, the principle of measurement and calibration is shown. The extracting of complex permeability from S-parameters is managed based on accomplished formulas, user-friendly program for computer control has been written, and obtained results are explained in detail. In order to verify proposed method, the results of the measurement of NiZn ferrite samples, manufactured by MMG NeoSid, are compared with catalog characteristics.

PACS numbers: 75.50.-y, 07.55.-w

\section{Introduction}

Magnetic permeability measurements are reviewed from the viewpoint of radio and communications applications. Concept of this parameter may be applied in circuit design and wave transmission calculations. Important radio and microwave magnetic materials are mainly thin films and nonconductor forms of powdered-iron suspensions and ferrites. The NiZn ferrites have wide usage in the industry. These materials have many useful properties, are versatile, and require many measurements for their characterization. There is large number of papers which represent demagnetizing effect, the effect of stress and the temperature effect of NiZn ferrite, [1-3]. The intrinsic complex permeability is the critical parameter for the optimization design, especially in the high-frequency applications.

Magnetic permeability is the ratio of magnetic flux density $\boldsymbol{B}$ to the applied magnetizing field $\boldsymbol{H}$ and it describes the interaction of a material with a magnetic field. Relative complex permeability $\tilde{\mu}_{\mathrm{r}}=\mu_{\mathrm{r}}^{\prime}-\mathrm{j} \mu_{\mathrm{r}}^{\prime \prime}$ consists of the real part $\mu_{\mathrm{r}}^{\prime}$ that presents the energy storage term and imaginary part $\mu_{\mathrm{r}}^{\prime \prime}$ that presents the power dissipation term. A number of papers have presented many different measurement techniques for characterization of magnetic materials such as free-space techniques, [4-5], waveguide techniques, [58], and coaxial line techniques, [9-10]. The waveguide techniques do not suffer radiation loss like free-space techniques and provide very accurate results. However, each waveguide has a limited frequency band of operation. High frequency measurement of complex permeability is based mainly on the coaxial

\footnotetext{
* corresponding author; e-mail: vasarad@uns.ac.rs
}

line method. Coaxial line method, that requires special construction of test sample (toroidal), gives accurate results and allows measurement in a very wide frequency range.

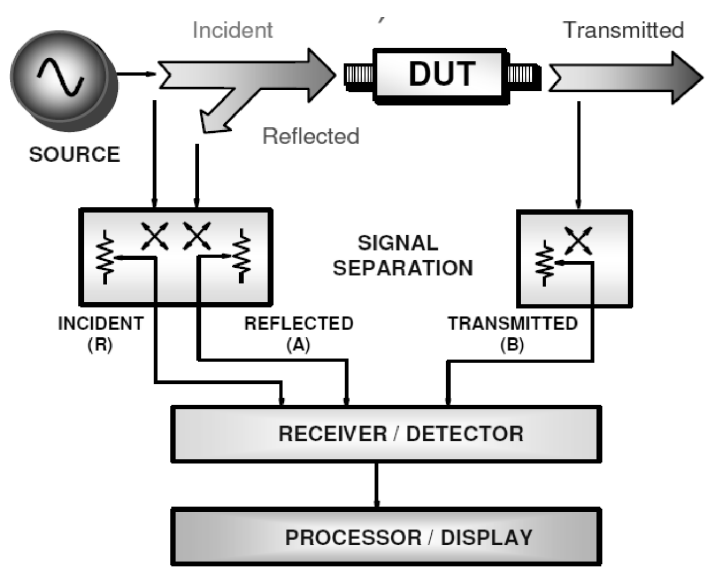

Fig. 1. Measurement principle of Agilent Technology E5071B network analyzer.

In order to determine the complex permeability of toroidal-shape samples, in this paper, measurements of S-parameters have been performed using the E5071B Agilent Technology vector network analyzer, that works in the frequency range between $300 \mathrm{kHz}$ and $8.5 \mathrm{GHz}$. Generalized block diagram of network analyzer and measurement principle are depicted in Fig. 1. The complex reflection coefficient $\Gamma=\Gamma^{\prime}+\mathrm{j} \Gamma^{\prime \prime}$ is obtained by measuring the ratio of a reflected signal to the incident signal. Supplies stimulus system can sweep frequency or power. Traditional network analyzers have one signal source while modern have the option for two internal 
sources. Signal separation block separates incident and reflected signals. The directional bridge circuit or coupler is used as a detector to detect the reflected signal, and the analyzer equipped with the bridge is used to supply and measure the signals. Since this method makes it possible to measure reflection at the device under test (DUT), it is applicable in RF and microwave range. The measured values of reflection coefficient are automatically converted into corresponding values of the impedance in the concerned frequency range. The other measurement values such are resistance $R$, inductance $L$, permittivity or permeability are calculated from the values of the measured components $\left(\Gamma^{\prime}, \Gamma^{\prime \prime}\right)$. The permeability of the test sample can be obtained by measuring the input differences between the short coaxial sample holder loaded with and without the toroidal sample.

This paper presents the experimental technique for measuring the complex permeability of toroidal sample, based on short coaxial line method in the frequency range between $300 \mathrm{kHz}$ and $1 \mathrm{GHz}$. Two original coaxial line holders were used from Agilent, the type 04191-85302 for small and the 16091-60012 for large samples. In comparison with some other coaxial techniques that are widely used, [10], proposed method does not require elimination of undesirable influences, such are residual impedance error and phase shift compensation. A simple method for high frequency measurement of S-parameter of toroidal magnetic samples has been designed and verified. In order to verify the proposed method, the results of NiZn ferrite samples measurements are compared with catalog results and have been discussed. Two NiZn samples (F14 and F19 ferrite) from MMG NeoSid, now TT electronic, have been used to verify the relevance of proposed method. In order to simplify the evaluation of obtained results and calculation process, a user-friendly program for computer control has been written.

\section{Measured principle}

High frequency measurement based on the coaxial line method allows to measure in a wide frequency range. For this purpose two different coaxial line holders have been used (Fig. 2). The first one was for large core (up to $10 \mathrm{~mm}$ ) and the other one was the original $50 \Omega$ construction for small cores (up to $7 \mathrm{~mm}$ ). The both holders are terminated with the APC-7 connector. Main characteristics of the given holders are summarized in Table I. The vector analyzer has a test port equipped with a fixed APC-7 connector. Sample holders consist of conductive shield surrounding the central conductor, which terminates in short circuit. The short circuit produces maximum of magnetic field and minimum of electric field near the sample, thus making the short circuit technique particularly suited for the measurement of the magnetic properties, such as permeability of the test sample. The medium between the inner and outer conductors of the cell is air. The inner height, $b$ is $20 \mathrm{~mm}$ for both holders, which obeys the condition $b<\lambda / 4$ for frequency $f \leq 1 \mathrm{GHz}$ in order to avoid the $\lambda / 4$-size resonance effect.

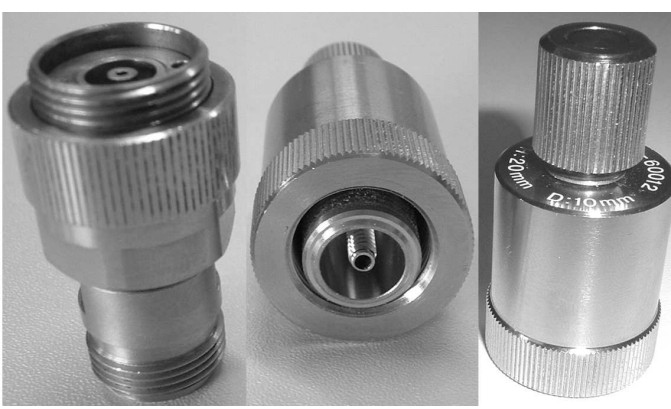

Fig. 2. APC-7 connector and standard short-coaxial line holder.

TABLE I

Main characteristics of short coaxial line holders.

\begin{tabular}{c|c|c}
\hline \hline Type & Impedance & Dimensions \\
\hline 04191-85302 & $Z_{0}=50 \Omega$ & $2 a=7 \mathrm{~mm}$ \\
& & $2 a_{1}=3 \mathrm{~mm}$ \\
& & $b=20 \mathrm{~mm}$ \\
\hline 16091-60012 & $Z_{0}=66 \Omega$ & $2 a=10 \mathrm{~mm}$ \\
& & $2 a_{1}=3 \mathrm{~mm}$ \\
& & $b=20 \mathrm{~mm}$
\end{tabular}

When the sample is inserted into the holder, the whole system is completely closed and then connected through the APC-7 to the previously calibrated network analyzer. The coaxial line supplies an electromagnetic wave propagating in a transverse electromagnetic (TEM) mode. The coaxial line can also support transverse electric (TE) and transverse magnetic (TM) modes in addition to a TEM mode. In practice, these modes are usually evanescent, and so have only reactive effect near discontinuities or sources where they are excited. Higher order modes will not appear if the sample length is less than one-half guided wave length of the fundamental mode. In this paper, the influences of the TE and TM modes in the measured frequency range are neglected.

The reflection coefficient is measured, permitting the determination of the input impedance of the cell with sample. Equation for the determination of complex permeability of the holder equipped with the test sample is derived in this section. Since the construction of this holder creates one turn around the toroid, Fig. 3, the complex magnetic flux $\tilde{\Phi}$, of the measurement circuit including the ring core is given by the equation:

$$
\tilde{\Phi}=\iint_{S} \boldsymbol{B} \mathrm{d} \boldsymbol{S}=\int_{0}^{a} \int_{0}^{b} \frac{\mu_{0} \mu_{\mathrm{r}} I}{2 \pi x} \mathrm{~d} x \mathrm{~d} y,
$$

where $\boldsymbol{B}$ presents complex phasor-vector of magnetic density, $\mu_{0}$ presents permeability of free space, $\mu_{\mathrm{r}}$ is relative permeability of sample, and $I$ is a complex phasor of harmonic time-dependent electrical current $i(t)$. By dividing the surfaces of cross section into the holder, the Eq. (2.1) for complex flux density is given by: 


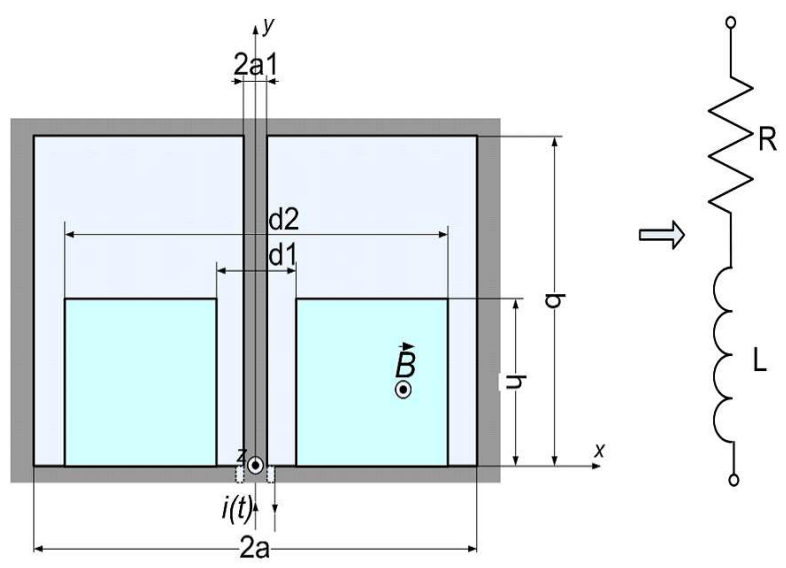

Fig. 3. Cross section of sample holder with toroidal sample and adequate electrical model.

$$
\begin{aligned}
\tilde{\Phi} & =\int_{\frac{d_{2}}{2}}^{a} \int_{0}^{b} \frac{\mu_{0} I}{2 \pi x} \mathrm{~d} x \mathrm{~d} y+\int_{\frac{d_{1}}{2}}^{\frac{d_{2}}{2}} \int_{0}^{h} \frac{\mu_{0} \tilde{\mu}_{\mathrm{r}} I}{2 \pi x} \mathrm{~d} x \mathrm{~d} y \\
& +\int_{\frac{d_{1}}{2}}^{\frac{d_{2}}{2}} \int_{h}^{b} \frac{\mu_{0} I}{2 \pi x} \mathrm{~d} x \mathrm{~d} y+\int_{0}^{\frac{d_{1}}{2}} \int_{0}^{b} \frac{\mu_{0} I}{2 \pi x} \mathrm{~d} x \mathrm{~d} y \\
& +\int_{0}^{a_{1}} \int_{0}^{b} \frac{\mu_{0} I}{2 \pi x} \mathrm{~d} x \mathrm{~d} y .
\end{aligned}
$$

The magnetic flux of measured circuit is then:

$$
\tilde{\Phi}=\frac{\mu_{0} I}{2 \pi}\left[\left(\tilde{\mu}_{\mathrm{r}}-1\right) h \ln \left(\frac{\mathrm{d}_{2}}{\mathrm{~d}_{1}}\right)+b \ln \left(\frac{a}{a_{1}}\right)\right],
$$

and the complex susceptibility, $\tilde{\chi}$, of a sample under test is given by equation:

$$
\tilde{\chi}=\frac{2 \pi\left(\tilde{\Phi}-\tilde{\Phi}_{\mathrm{air}}\right)}{h I \mu_{0} \ln \left(\frac{\mathrm{d}_{2}}{\mathrm{~d}_{1}}\right)},
$$

where $\tilde{\Phi}_{\text {air }}$ is a magnetic flux when ferrite cores is not mounted into the holder:

$$
\tilde{\Phi}_{\text {air }}=\frac{b \mu_{0} I}{2 \pi} \ln \left(\frac{a}{a_{1}}\right) .
$$

The measured complex impedance, $Z$, of an equivalent electrical circuit of the cell loaded with the ferrite core, shown in Fig. 3 can be defined as $\tilde{Z}=R+\mathrm{j} \omega L=\mathrm{j} \omega \tilde{\Phi} / I$. Instead of fluxes $\tilde{\Phi}$ i $\tilde{\Phi}_{\text {air }}$ in Eq. (2.4) we can use corresponding complex impedance $Z$ and $Z_{\text {air }}$, measured with and without magnetic core, respectively:

$$
\tilde{\mu}_{\mathrm{r}}=1+\tilde{\chi}=1+\frac{\left(\tilde{Z}-\tilde{Z}_{\text {air }}\right)}{\mathrm{j} h \mu_{0} f \ln \left(\frac{\mathrm{d}_{2}}{\mathrm{~d}_{1}}\right)},
$$

where $d_{1}$ and $d_{2}$ denotes the inner and outer diameters of the toroid, respectively, $h$ is the height of the toroid and $f$ is the frequency of applied $a c$ electromagnetic field. Complex permeability is therefore calculated from the difference between the impedance of holder loaded with and without toroidal sample. The vector analyzer measures the complex reflection coefficient, which is recalculated to the input impedance of the cell (with or without sample) according to the following equation:

$$
\tilde{Z}_{\text {in }}=Z_{0} \frac{1+\tilde{\Gamma}}{1-\tilde{\Gamma}},
$$

with $Z_{0}=50 \Omega$ characteristic impedance of the $7 \mathrm{~mm}$ test port. Resistance $R_{\text {in }}$ and reactance $X_{\text {in }}$ values of the input impedance of the cell $\tilde{Z}_{\text {in }}=R_{\text {in }}+\mathrm{j} X_{\text {in }}$ can then be calculated using the following relations:

$$
\begin{aligned}
& R_{\text {in }}=Z_{0} \frac{1-\Gamma^{\prime 2}-\Gamma^{\prime \prime 2}}{\left(1-\Gamma^{\prime}\right)^{2}+\Gamma^{\prime \prime 2}}, \\
& X_{\text {in }}=Z_{0} \frac{2 \Gamma^{\prime \prime}}{\left(1-\Gamma^{\prime}\right)^{2}+\Gamma^{\prime \prime 2}} .
\end{aligned}
$$

Since $Z_{\text {in }}$ of the holder with and without toroidal sample is known, the complex (relative) permeability $\tilde{\mu}_{\mathrm{r}}$ is obtained by Eq. (2.6).

Tangent loss factor, $\operatorname{tg} \delta$, can be calculated as a difference between imaginary and real parts of complex permeability:

$$
\operatorname{tg} \delta=\frac{\mu_{\mathrm{r}}^{\prime \prime}}{\mu_{\mathrm{r}}}
$$

and relative loss factor, L.F. can be obtained using following equation:

$$
L . F .=\frac{\operatorname{tg} \delta}{\mu_{i}},
$$

where $\mu_{i}$ is the initial permeability of samples.

\section{Experimental results}

In order to verify the proposed method that uses short coaxial line holder, two test samples of MMG NeoSid are used, based on NiZn material called F14 and F19. The photograph of test samples is shown in Fig. 4. The basic characteristics of used samples are shown in Table II where $B_{\mathrm{s}}$ denotes saturation flux density, $B_{\mathrm{r}}$ is remanent flux density, $H_{\mathrm{c}}$ is coercitivity. Dimensions of both samples are shown in Table II The thickness of samples satisfies the condition $h<\lambda / 4$, at which dimensional resonance effect cannot occur in the measured results.

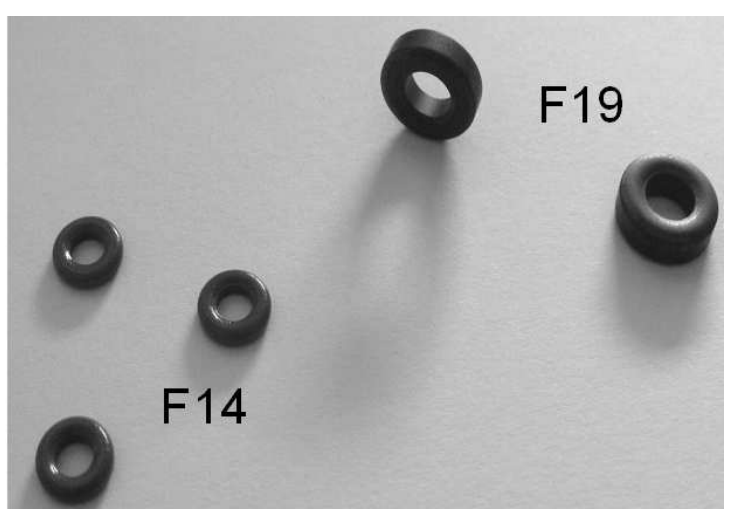

Fig. 4. Photograph of test samples: F14 and F19.

Calculated results obtained using proposed equation for real and imaginary parts of complex permeability for 


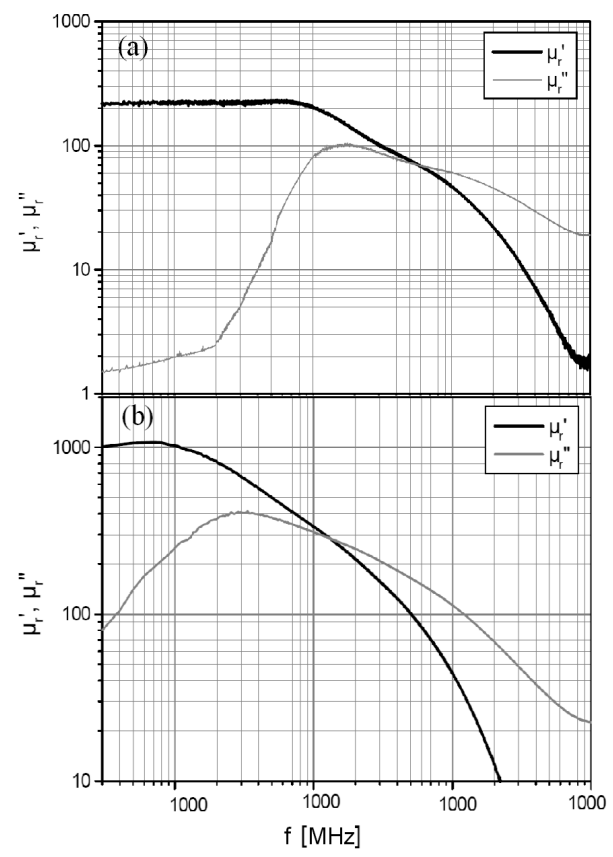

Fig. 5. Calculated results for real and imaginary part of complex permeability for: (a) F14, (b) F19 samples.

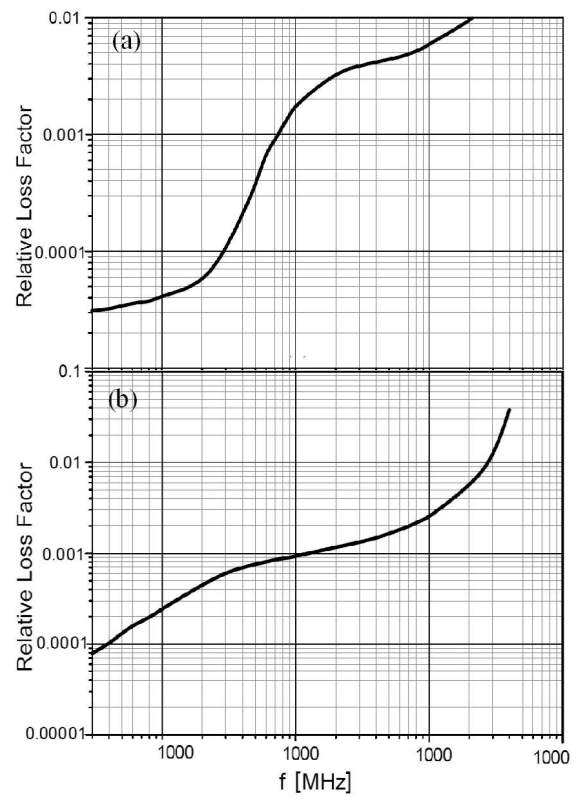

Fig. 6. Calculated results for relative loss factor for: (a) F14, (b) F19.

F14 and F19 samples in function of frequency are shown in Fig. 5. Figure 6 presents calculated relative loss factor for measured samples. All measurements are done in an ideal condition at the temperature of $25^{\circ} \mathrm{C}$. The change of real part of complex permeability with frequency close to the critical frequency $f_{\mathrm{c}}$ is called the dispersion of permeability and the change of imaginary part with frequency is known as the absorption. Sintered NiZn ferrites
TABLE II

Characteristics parameters of MMG NeoSid samples: F14 and F19.

\begin{tabular}{l|c|c}
\hline \hline & $\mathrm{F} 14$ & $\mathrm{~F} 19$ \\
\hline Dimensions $[\mathrm{mm}]$ & $6.35 \times 3.18 \times 1.52$ & $9.52 \times 4.75 \times 3.18$ \\
\hline Initial permeability & 220 & 1000 \\
& $B<0.1 \mathrm{mT} / 10 \mathrm{kHz}$ & $B<0.1 \mathrm{mT} / 10 \mathrm{kHz}$ \\
$B_{\mathrm{S}}[\mathrm{mT}]$ & 350 & 260 \\
$B_{\mathrm{r}}[\mathrm{mT}]$ & 217 & 165 \\
$H_{\mathrm{c}}[\mathrm{A} / \mathrm{m}]$ & 172 & 53
\end{tabular}

showed a resonant type of frequency dispersion. At low frequency, NiZn samples have high values of real part of complex permeability (at about 200 for F14 and about 1000 for F19). These characteristics attributed to the natural resonance of spin rotation and the vibrational resonance of magnetic domain walls and they are not monotonous. It is well known that permeability spectra of the ferrites can be decomposed into spin rotational component and the domain wall component [1]. The spin rotational component is of relaxation type (due to the large damping factor of the spin rotation in the ferrite) and its dispersion is inversely proportional to the frequency. The domain wall component is of resonance type and depends on the square of the frequency. The shape of ferrite characteristics depends on these resonances that depend on the temperature, stress and chemical composition of ferrite. Critical resonant frequency, $f_{\mathrm{c}}$ is the value at which the imaginary part has maximum and for F14 and F19 this frequency is equal to $25 \mathrm{MHz}$ and $3 \mathrm{MHz}$, respectively. It is proportional to the saturation magnetization of ferrite filler and with arising demagnetization fields of ferrite particles.

In order to verify proposed method, obtained measurement results for NiZn ferrite samples are compared with the catalog characteristics. Eligible correspondence between results extracted using short coaxial line method and catalog characteristics, is obtained in the measured frequency range. Small variations exist as a consequence of tolerance of sample dimensions.

\section{Discussion}

Over the years, there has been a number of methods developed for measuring electromagnetic permeability. These techniques include free-space methods, waveguide techniques, open-ended coaxial-probe techniques, cavity resonators, and dielectric-resonator techniques. Each method has its range of applicability and its inherent limitations. For example, techniques based on cavities are accurate, but not broadband, and are usually limited to low-loss materials. The waveguide techniques do not suffer from radiation loss like free-space techniques and provide very accurate results. However, each waveguide has a very limited frequency band of operation. Coaxial line method gives accurate results and allows measurement in the very wide frequency range. 
In this paper, experimental one-port technique for measuring the complex permeability based on short coaxial line method is proposed. The short-circuit line method is a fast, sensitive and accurate broadband measurement technique. In this approach, measurements are obtained by placing a toroidal sample in the coaxial line terminated in short-circuit and measuring the change in S-parameters compared to a measurement in free space using a network analyzer.

In comparison with some other short-coaxial techniques that are widely used, proposed method does not require elimination of undesirable influences, such are residual impedance error and phase shift compensation. A limitation of this technique is that it requires cutting of the sample and therefore this technique does not fall under the general category of nondestructive testing method. Proposed one-port measurement is useful when two-port measurements are not possible, for example, in high temperature measurements and remote sensing applications.

The main aim of this paper is developing of the user-friendly program for computer control and measurement process. The program allows to: define the measurement conditions (frequency range, frequency step, dimensions, etc.), recalculate the measured values to the corresponding values of complex permeability, store the measured data and export them in a format convenient for Ansoft HFSS electromagnetic simulator or SPIS simulator. The change of magnetic material properties versus frequency is very important parameter for future simulations, wave transmission calculations and circuit design.

\section{Conclusion}

In this paper, short circuit sample holder for measuring a complex permeability of toroidal magnetic samples is described. The measurement principle of system that allows measurement of complex permeability in wide frequency range is proposed. Special attention has been paid to conversion measured values of complex reflection coefficients to the characteristic impedance. Program code for computer control processing of measuring results is written. In order to verify proposed method, the results of measurements are compared with catalog characteristics for NiZn samples F14 and F19. A good arrangement with results obtained using short coaxial line method can be obtained in the wide frequency range, except for shift in a frequency that is a consequence of tolerance of sample dimensions and characteristics.

\section{References}

[1] T. Tsutaoka, M. Ueshima, T. Tokunaga, J. Appl. Physics 78, 3983 (1995).

[2] M. Ledieu, O. Acher, J. Magn. Magn. Mater. 258, 144 (2003).

[3] F. Fiorillo, C. Beatrice, M. Coïsson, Lj. Zhemchuzhna, IEEE Trans. Mag. 45, 4242 (2009).

[4] D.K. Ghodgaonkar, V.V. Varadan, V.K. Varadan, IEEE. Trans. Instum. Meas. 39, 387 (1990).

[5] J.B. Jarvis, M.D. Janezic, B.F. Riddle, R.T. Johnk, R. Kabos, C.L. Holloway, R.G. Geyer, C.A. Grosvenor, NIST Technical Note 1536, Boulder, CO 2005, p. 1536.

[6] B. Jarvis, M.D. Janezic, C.A. Grosvenor, R.G. Geyer, NIST Technical Note 1355, Boulder, CO 1993, p. 1355.

[7] N.N. Al-Moyaed, M.N. Afsar, U.A. Khan, S. McCooey, M. Obol IEEE Trans. Mag. 44, 1768 (2008).

[8] K. Chalapat, K. Sarvla, J. Li, G.S. Paraoanu, IEEE T. Microw. Theory 57, 2257 (2009).

[9] R. Huang, D. Zhang, IEEE Trans. Magn. 44, 597 (2008).

[10] R. Dosoudil, E. Ušak, V. Olah, Journal of Electrical Engineering 57, 105 (2006). 